\title{
Differential Modulation of Platelet Adhesion and Spreading by Adhesive Ligand Density
}

\author{
Revital Zarka, ${ }^{\dagger, \text { II }}$ Melanie B. Horev, ${ }^{\dagger, I I}$ Tova Volberg, ${ }^{\dagger}$ Stefanie Neubauer, ${ }^{\ddagger}$ Horst Kessler, ${ }^{\ddagger}$ \\ Joachim P. Spatz, ${ }^{\S \odot}$ and Benjamin Geiger, ${ }^{* \dagger}$ \\ ${ }^{\dagger}$ Department of Molecular Cell Biology, The Weizmann Institute of Science, Rehovot 76100, Israel \\ ${ }^{\ddagger}$ Institute for Advanced Study (IAS) and Center of Integrated Protein Science, Department of Chemistry, Technical University of \\ Munich, 85747 Garching, Germany \\ ${ }^{\S}$ Department of Cellular Biophysics, Max Planck Institute for Medical Research, Jahnstrasse 29, D-69120 Heidelberg, Germany \\ Department of Biophysical Chemistry, University of Heidelberg, D-69120 Heidelberg, Germany
}

\section{Supporting Information}

\begin{abstract}
Platelets play a major role in hemostasis and thrombosis, by binding to the underlying extracellular matrix around injured blood vessels, via integrin receptors. In this study, we investigated the effects of adhesive ligand spacing on the stability of platelets' adhesion and the mode of their spreading on extracellular surfaces. Toward this end, we have examined the differential adhesion and spreading of human platelets onto nanogold-patterned surfaces, functionalized with
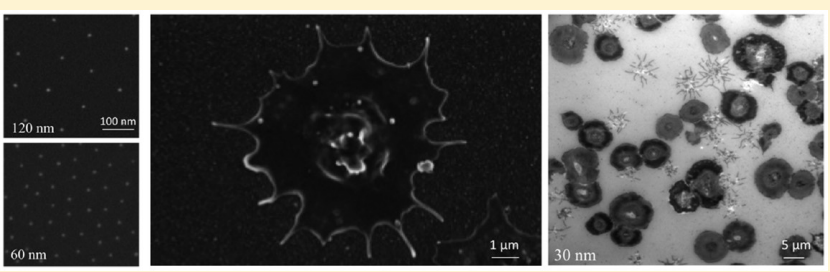
the $\alpha \mathrm{IIb} \beta 3$ integrin ligand, SN528. Combining light- and scanning electron-microscopy, we found that interaction of platelets with surfaces coated with SN528 at spacing of 30-60 nm induces the extension of filopodia through which the platelets stably attach to the nanopatterned surface and spread on it. Increasing the nanopattern-gold spacing to $80-100 \mathrm{~nm}$ resulted in a dramatic reduction (>95\%) in the number of adhering platelets. Surprisingly, a further increase in ligand spacing to $120 \mathrm{~nm}$ resulted in platelet binding to the surface at substantially larger numbers, yet these platelets remained discoid and were essentially devoid of filopodia and lamellipodia. These results indicate that the stimulation of filopodia extension by adhering platelets, and the consequent spreading on these surfaces depend on different ligand densities. Thus, the extension of filopodia occurs on surfaces with a ligand spacing of $100 \mathrm{~nm}$ or less, while the sustainability and growth of these initial adhesions and induction of extensive platelet adhesion and spreading requires lower ligand-to-ligand spacing $(\leq 60 \mathrm{~nm})$. The mechanisms underlying this differential ligand-density sensing by platelets, as well as the unexpected retention of discoid platelets on surfaces with even larger spacing $(120 \mathrm{~nm})$ are discussed.
\end{abstract}

KEYWORDS: Platelets, adhesion, nanopatterned surfaces, integrin ligand

$\mathrm{P}$ latelets are cell fragments, derived from bone marrow megakaryocytes, which play an essential role in arresting bleeding from injured vessels. ${ }^{1,2}$ They are involved in multiple physiological processes, including the regulation of vascular integrity, angiogenesis, inflammation, and wound healing. ${ }^{3}$ It had been extensively demonstrated that the functionality of platelets is tightly regulated by their selective adhesion processes, whereby they adhere to sites of injury, where endothelial cells are altered, the subendothelial extracellular matrix (ECM) is exposed, and a blood clot is formed. ${ }^{4-7}$ Initial adhesive interaction with extracellular surfaces, such as a fibrin clot (mediated via $\alpha \mathrm{IIb} \beta 3$ integrin receptors) or basement membrane-associated collagen (via $\alpha 2 \beta 1$ integrin or GP VI), trigger platelet activation, which further regulates the adhesion to and spreading on the external surfaces. ${ }^{8,9}$ These, and additional, postinjury processes, like the binding of von Willebrand factor to collagen, modulate the adhesion specificity and exert major effects on platelet structure and cytoskeletal organization. $^{10-14}$
Following initial interactions with the extracellular matrix (ECM), discoid platelets undergo activation, manifested by the extension of multiple filopodia that mediate the initial adhesion to the surface. Depending on the nature of the surface, the presence of specific activation agonists and the mechanical environmental conditions (e.g., shear stress), platelets can enhance their adhesion to the surface and spread on it. ${ }^{15-17}$ Actin filament assembly serves as the primary driving force for the extension of filopodia and lamellipodia, and actin 'nodules' were described, and suggested to play a role in the mechanocoupling between platelets and the substrate. ${ }^{18}$ That said, the mechanism underlying the initial, actin-driven stages of adhesion and spreading are still poorly understood. It was shown that ligand density affect integrin mediated cell adhesion. ${ }^{19}$ It was proposed that upon initial contacts of

Received: August 30, 2018

Revised: January 8, 2019

Published: January 16, 2019 

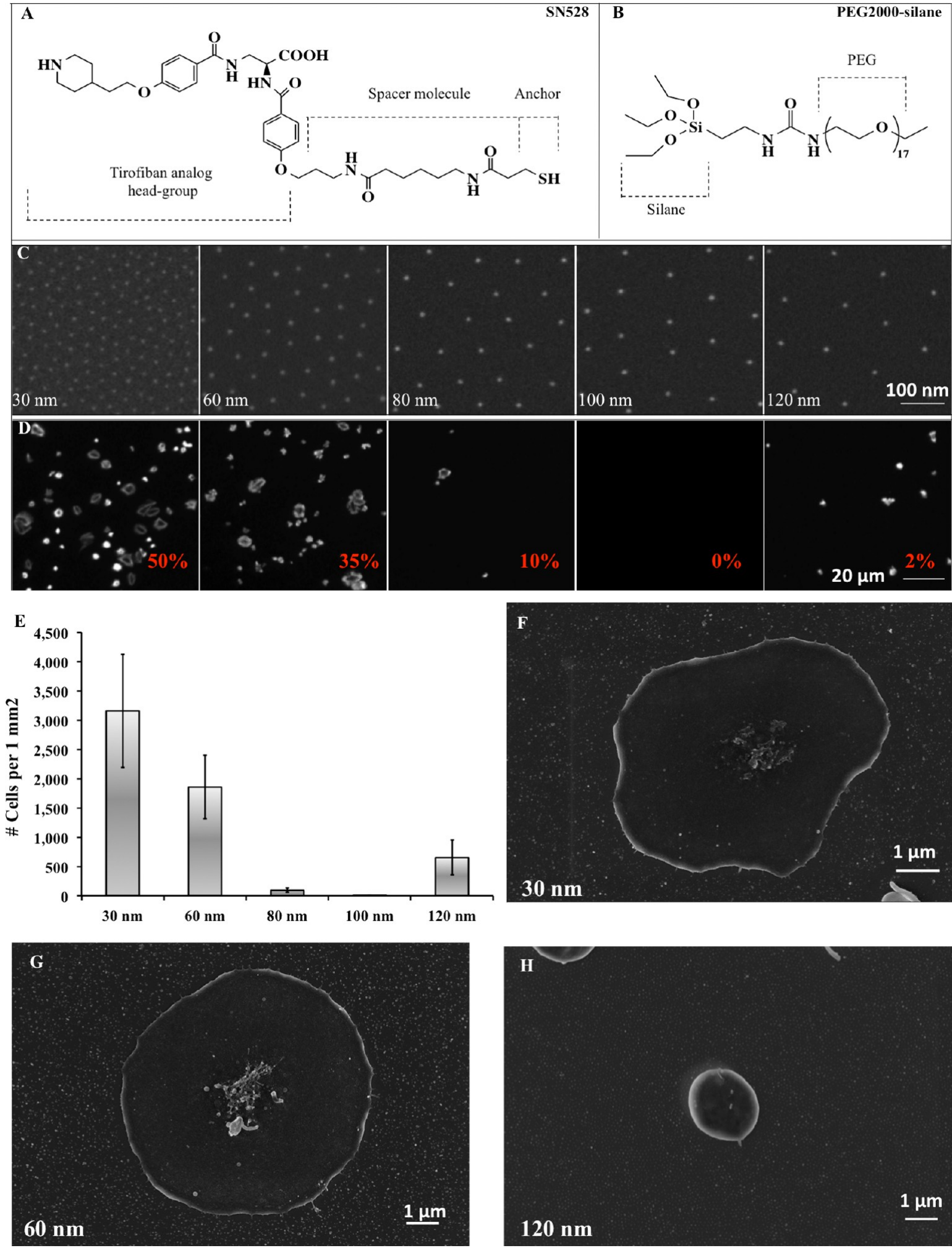

Figure 1. (A) Structure of SN528, a mimetic ligand of $\alpha \mathrm{IIb} \beta 3$ integrin. Chemical binding to the nanogold occurs via the SH group; (B) PEG2000silane, which binds the exposed glass surface, and serves as cell repellant layer; (C) Representative HR-SEM micrograph of nanostructured glass surfaces with interparticle spacing of 30,60,80,100, and $120 \mathrm{~nm} \pm 10$; (D) Immunofluorescence images of actin-labeled platelets seeded on (left to right) 30,60,80,100, and $120 \mathrm{~nm}$ nanopatterned surfaces, bioactivated with SN528; (E) Quantification of the number of platelets per $1 \mathrm{~mm}^{2}$ of substrate. The statistical difference between the platelets attaching to 30 and $60 \mathrm{~nm}$ surfaces determined by $t$ test was $p<0.01$, and the difference between 30 and $60 \mathrm{~nm}$ surfaces to all other surfaces determined by ANOVA test was $p<0.001$. The results were checked by the Kolmogorov-Smirnov test. Standard deviation is displayed; $(\mathrm{F}-\mathrm{H})$ SEM images of platelets adhering to surfaces with 30, 60, and $120 \mathrm{~nm}$ nanogold spacing.

$\alpha \operatorname{IIb} \beta 3$ integrin with surface-bound fibrinogen or fibrin, a kinase-signaling cascade is activated, which enhances the adhesion and spreading process, ${ }^{20}$ orchestrated by actin modulators, such as gelsolin, $\beta 4$-thymosin, and profilin. ${ }^{21}$ 

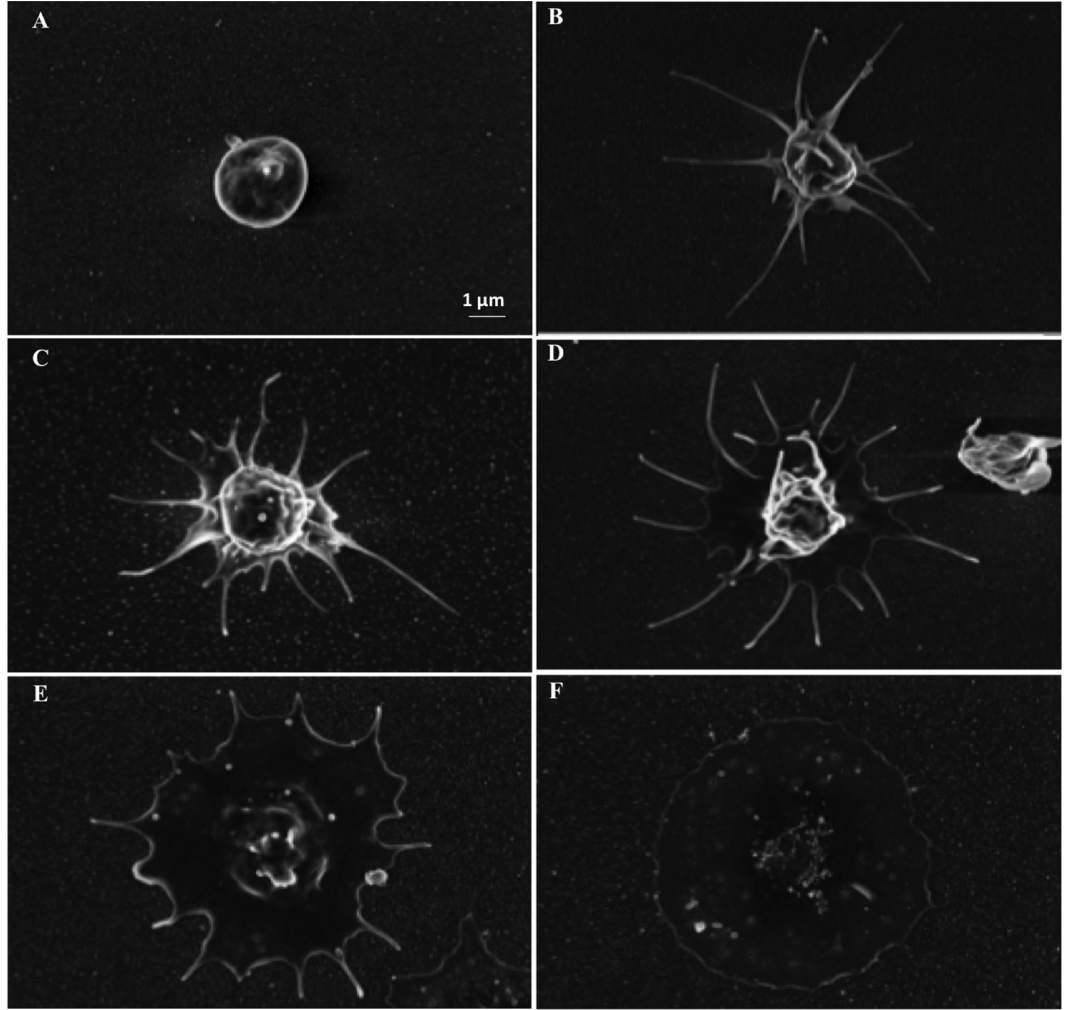

\begin{tabular}{|l|c|c|c|c|c|c|}
\hline Image & A & B & C & D & E & F \\
\hline Roughness & 1.00 & 1.45 & 1.73 & 1.61 & 1.49 & 1.06 \\
\hline
\end{tabular}

Figure 2. SEM images of platelet spreading on $60 \mathrm{~nm}$ surface, showing (A) A discoid platelet, demonstrating the primary contact; (B-C). Platelet with filopodial extensions. (D-E) Platelets with lamellipodial spreading; (F) Fully spread platelet; (G).Calculated perimeter-to-convex hall to perimeter ratio, indicating the roughness of the platelets' perimeter. These values are representative values of each phase to illustrate the visual representation and to show a qualitative measure to highlight the differences.

Further cytoskeletal reorganization triggers the secretion of platelet agonists such as adenosine diphosphate (ADP) and serotonin, as well as adhesive proteins such as platelet factor 4, fibrinogen, and von Willebrand factor. ${ }^{20,22}$ Once secreted, these agonists induce further cytoskeletal reorganization, leading to Rac1- and $\alpha \mathrm{IIb} \beta 3$-dependent lamellipodia formation and assembly of contractile actin bundles. ${ }^{23,24}$

Throughout this process, platelets apply force to the underlying surfaces, ${ }^{25,26}$ which induces the formation of focal adhesions (FA)-like structures. In their absence of contractile forces (e.g., following relaxation induced by myosin II or Rho kinase inhibitors) these adhesions undergo disassembly. ${ }^{27-30}$ Such forces, generated by adhering platelets, play important physiological roles, both in the reinforcement of the adhesion and in processes such as wound closure and clot retraction. ${ }^{31,32}$ It was further shown that the assembly of adhesion sites is regulated not only by the chemical composition of the substrate but also by its physical properties, including rigidity, ${ }^{33-36}$ microtopography, ${ }^{37-40}$ and ligand spacing at the micro- and nanoscales. ${ }^{41-47}$ It was further established that keeping ligand density constant while increasing substrate stiffness, results in stronger adhesion, ${ }^{48}$ yet in recent studies, ${ }^{49}$ it was shown that adhesion to soft surfaces can be maintained even when ligand spacing is large and insufficient for supporting adhesion to stiff substrates.
In this study, we address the effect of ligand spacing on the formation and stability of platelet adhesions to surfaces, functionalized with a biomimetic ligand (SN528), highly specific for $\alpha \mathrm{IIb} \beta 3$ integrin, with IC50 $=3.58 \mathrm{nM}^{50}$

To address this issue, human platelets were seeded on ordered nanopatterned surfaces, consisting of gold nanoparticles, spaced by $30-120 \mathrm{~nm}$, and functionalized by a high affinity $\alpha \operatorname{IIb} \beta 3$ integrin ligand, namely, SN528. Combining live cell microscopy, with immunofluorescence and scanning electron microscopy, we monitored the adhesion, spreading and cytoskeletal reorganization in adhering platelets. The results, presented here show that lateral spacing of integrin $\alpha \mathrm{IIb} \beta 3$ ligand of up to $60 \mathrm{~nm}$ supports platelet spreading and the formation of stable adhesions. Seeding platelets on surfaces with larger spacing yielded unexpected results, manifested by the essentially complete failure of the platelets to adhere to nanopatterned surfaces with 80 or $100 \mathrm{~nm}$ spacing, yet retaining their capacity to attach, without spreading, to surfaces with $120 \mathrm{~nm}$ ligand spacing. The implications of these results on the differential effects of ligand density on initial platelet activation and the formation of stable matrix adhesions are discussed.

To evaluate the effect of ligand spacing on platelets attachment and spreading, fresh human platelets were seeded on nanopatterned surface, functionalized with SN528 (Figure 


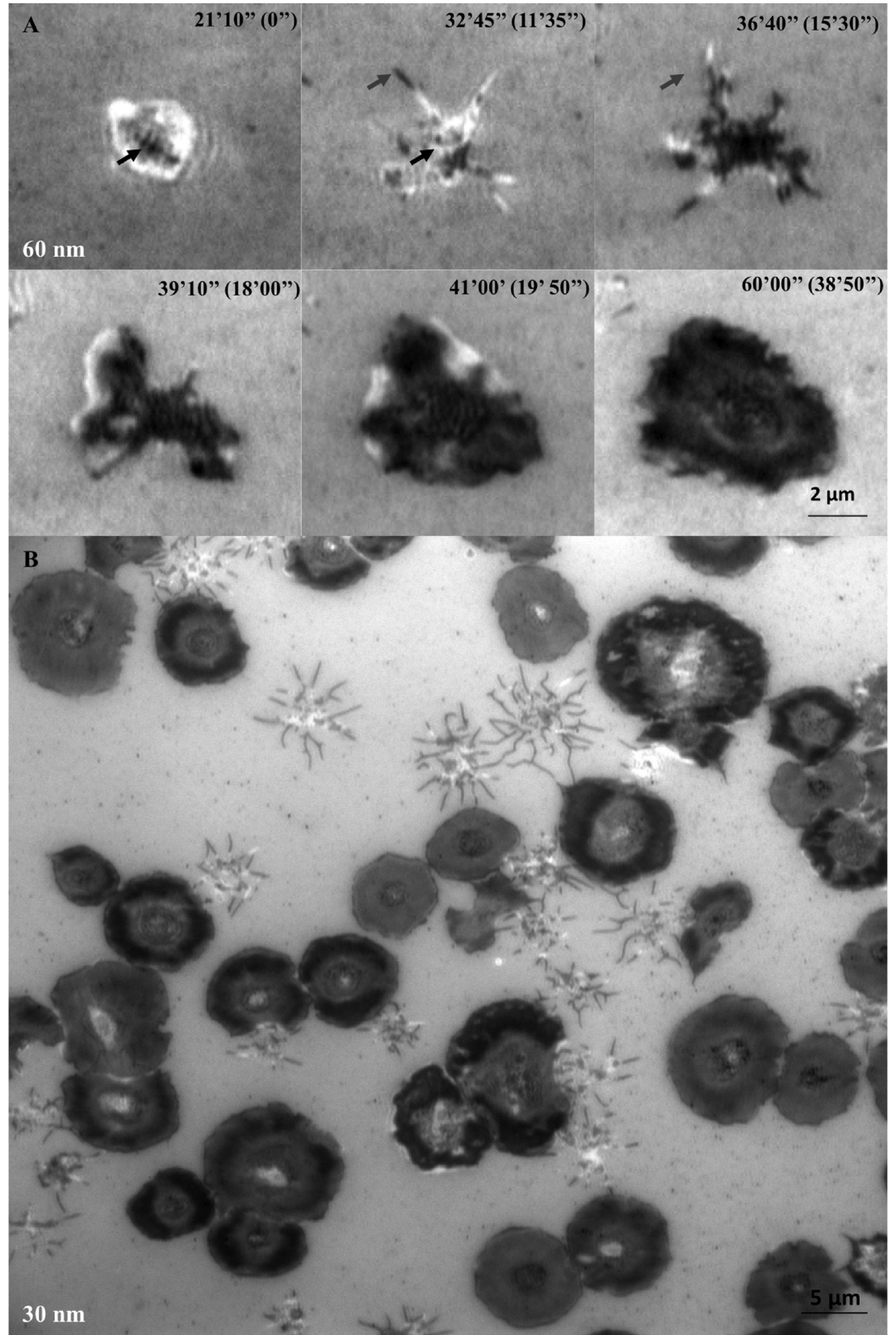

Figure 3. (A) IRM snapshots from live imaging of platelet on $60 \mathrm{~nm}$ spacing, showing different stages in the spreading process (left to right): primary contact; extension of filopodia, lamellipodial growth, fully spread platelet. Real time visible in upper right corner, and normalized time in parentheses. Black arrows indicated transient features from time point 1-2, and blue arrows indicate transient features from time point 2-3. (B) IRM image of platelets spreading on substrate with $30 \mathrm{~nm}$ spacing.

$1 \mathrm{~A}$ ), a peptidomimetic ligand of $\alpha \mathrm{IIb} \beta 3$ integrin (based on the antithrombotic agent Tirofiban ${ }^{51}$ ).

This mimetic ligand demonstrated superior specificity for platelet integrin $\alpha \mathrm{IIb} \beta 3$, compared to a variety of RGD peptides, ${ }^{50,52}$ in line with our experiments (data not shown). Prior to functionalization, the surfaces were passivated with PEG-silane (Figure 1B). The regularity of the nanogold patterns was verified using SEM imaging as shown in Figure 1C.

To evaluate the effect of interparticle spacing on adhesion and spreading, platelets were fixed, permeabilized, and visualized by F-actin labeling $1 \mathrm{~h}$ after plating. The results, based on five independent experiments, indicated that SN528-gold spacing has a dramatic effect on platelets attachment and spreading (Figure 1D). Platelets attached and spread best on surfaces with
$30 \mathrm{~nm}$ spacing as seen by SEM (Figure $1 \mathrm{~F}$ ), with moderately lower adhesion to surfaces with $60 \mathrm{~nm}$ spacing (Figure 1E), although still leading to the formation of fully spread platelets (Figure 1G). It is noteworthy that adhesion fo platelets to the densely patterned surfaces was comparable to the adhesion to surfaces, uniformly functionalized with fibrinogen (data not shown). Increasing the spacing to $80 \mathrm{~nm}$ resulted in over $95 \%$ decline in the number of attached platelets, and on the $100 \mathrm{~nm}$ substrates, only few, poorly spread cells were found (Figure 1D). Unexpectedly, further increasing the spacing to $120 \mathrm{~nm}$ significantly increased the number of attached cells, which were still significantly lower (25\% and $40 \%)$ than the number of platelets attached to the surfaces with 30 and $60 \mathrm{~nm}$ spacings, 


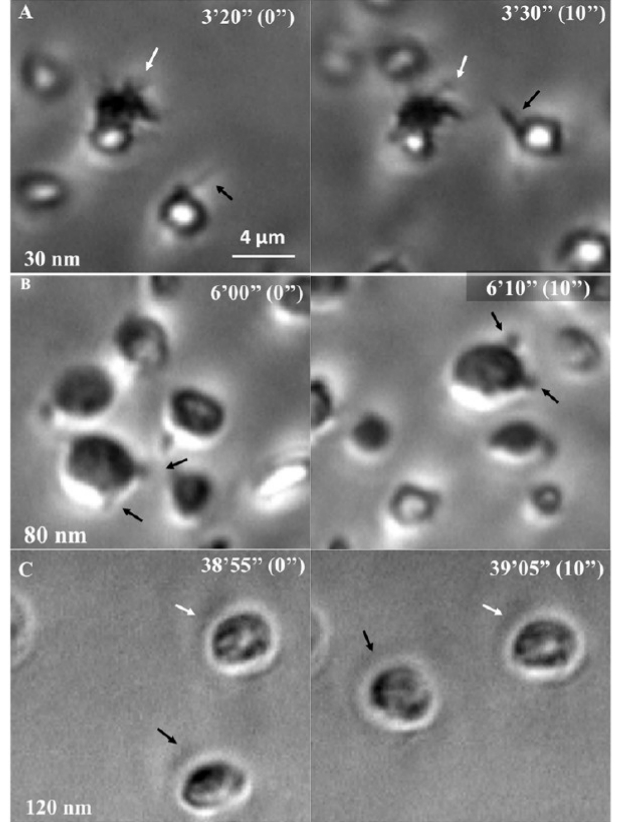

Platelets on Nanopatterned surfaces - time spent in each phase of adhesion

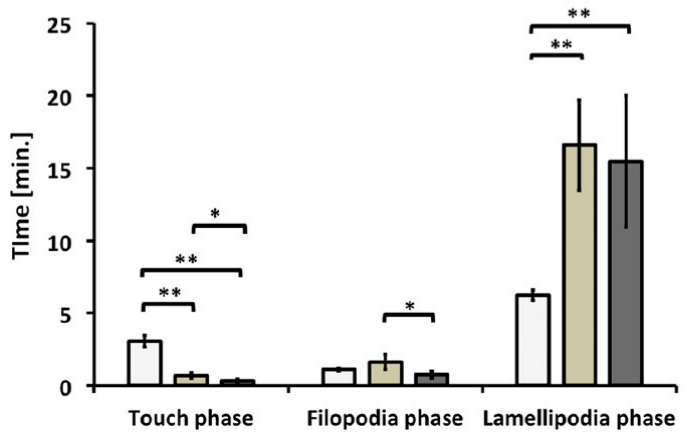

${ }^{* *} \mathbf{p}<0.00$

$* \mathrm{p}<0.005$

- Fibrinogen

$\square 30 \mathrm{~nm}$

$\square 60 \mathrm{~nm}$

Figure 4. Images of spreading platelets, acquired during live phase contrast imaging (10" intervals), demonstrating the dynamic interaction with the substrates in two subsequent frames (left to right). (A) Images acquired on $30 \mathrm{~nm}$ spacing, close to the surface, showing filopodial extensions (marked with black arrows) and platelet adhering via filopodia (white arrows); (B) Platelets located close to the $80 \mathrm{~nm}$ pattern, failing to adhere to the surface through filopodia (black arrows). (C) Platelets on $120 \mathrm{~nm}$ surfaces, mostly discoid, free-floating (black arrow), or adhering (white arrow); (A-C) Real time visible in upper right corner, and normalized time in parentheses. (D) Platelets on fibrinogen (control) and on 30, $60 \mathrm{~nm}$ patterned surfaces, indicating the time spent in each stage of adhesion (see text). Statistical test used: $t$ test, standard deviation is displayed.

respectively, and mainly exhibiting the discoid shape (Figure $1 \mathrm{H})$.

SEM examination of platelets, fixed at different stages of spreading, provided information on the morphological transition of platelets from discoid to filopodia-rich adherent stage (Figure 2A,B) and the progression to lamellipodial spreading (Figure 2C-F). This demonstrated that both the filopodia and the lamellipodia are extended at the ventral aspect of the platelets, in line with the interference reflection microscopy (IRM) monitoring (Figure 3A). The progressive transition from initial discoid adhesion to filopodial and lamellipodial spreading is manifested by increased "roughness" of the cells' perimeter, during the filopodial stage, and a reduction in roughness with the outward extension of the lamellipodium (Figure $2 \mathrm{G}$ ).
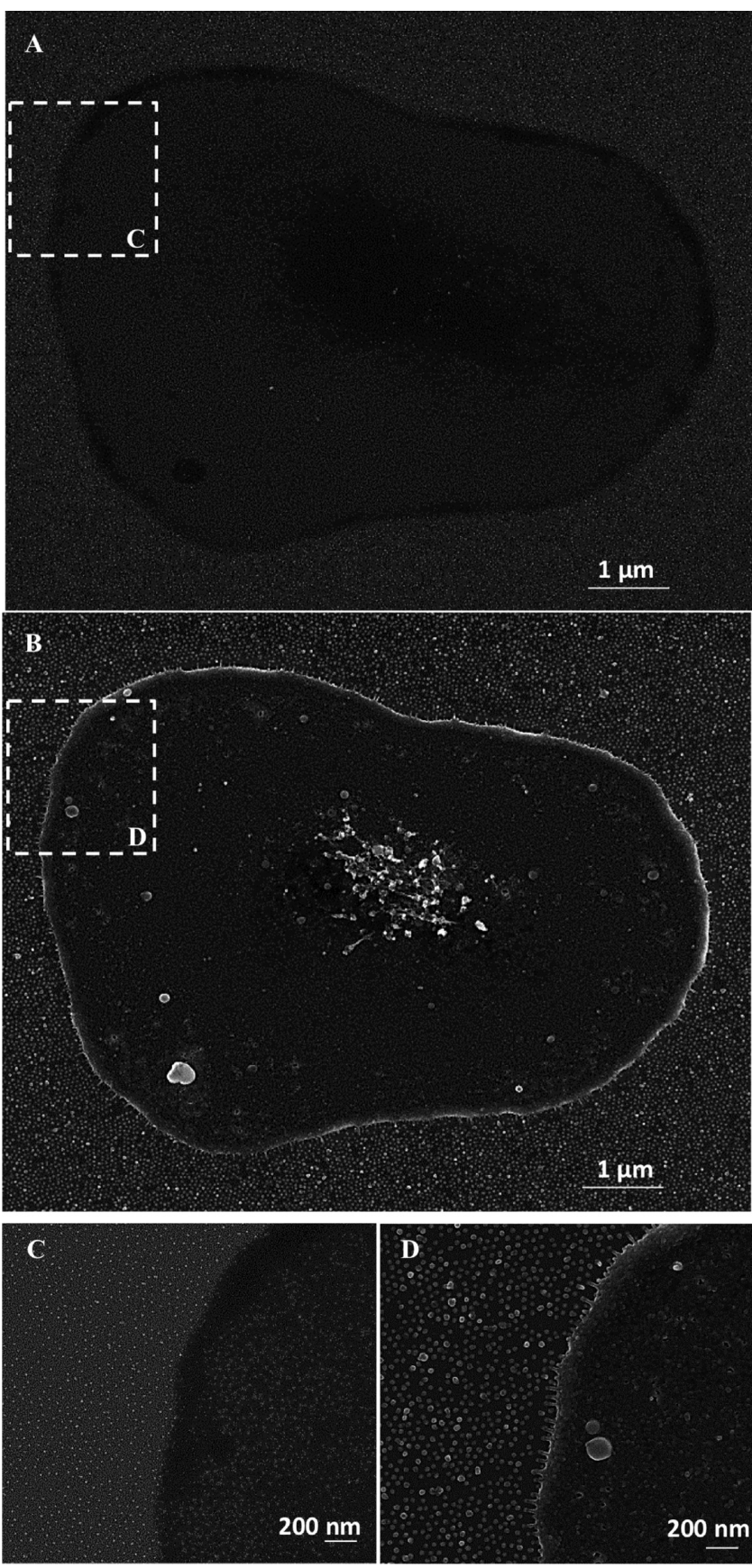

Figure 5. (A,B) High-resolution visualization of platelets spread on SN528-nanogold with $60 \mathrm{~nm}$ spacing, using a backscattered electron detector (BSE) and InLens detector, respectively. (C,D). Higher magnification micrograph of the same platelet imaged in A and B. Note the platelet's nanoprotrusions attached to individual particles.

To monitor the initial dynamics of the spreading process, we have compared, by real-time IRM (Figure 3 ) or phase-contrast optics (Figure 4), shape changes shortly after plating.

Live cell IRM (Figure 3, also see Movie S1) indicated that the earliest physical contacts with the substrate are transient, with short lifespan of seconds to a few minutes (Figure 3A, time points 1-3), followed by the formation of stable adhesions to the external surface, initially mediated via the tip or shaft of filopodia (Figure 3A, time points 2 to 4). Upon longer incubation, a fraction of the platelets (varies between 40 and $55 \%)$ lamellipodia are formed, which initially "fill in" the gaps 

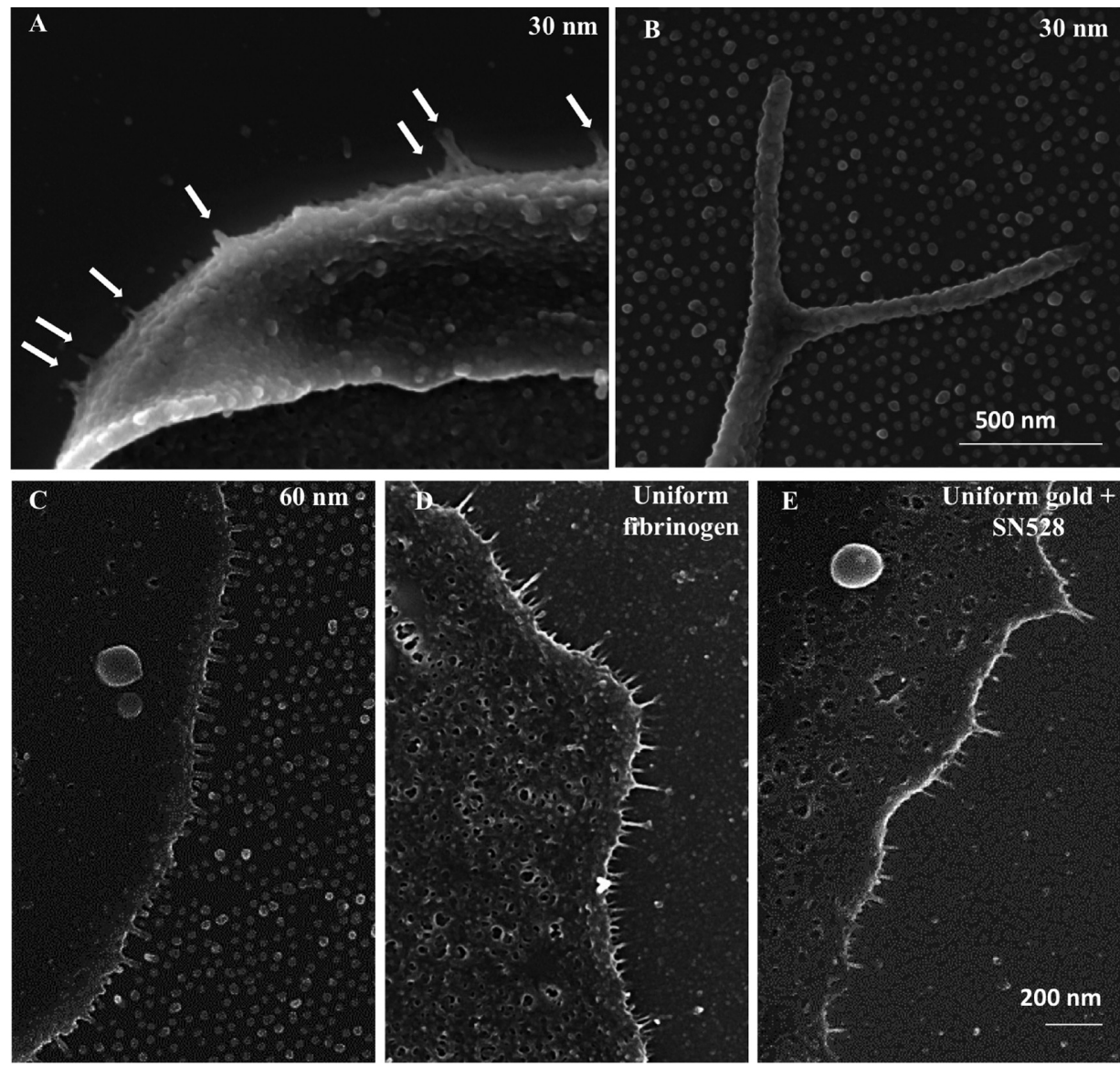

Figure 6. (A) SEM micrograph showing platelet adhering to the nanopatterned surface through short membrane nanoprotrusions (white arrows); (B) Filopodial extension of adhering platelet, associated with the nanopatterned surface through membrane nanoprotrusions interacting with the gold particles. (C-E). Left to right: Contact areas of platelets spread on $60 \mathrm{~nm}$ surface, homogeneous fibrinogen-coated, and gold/SN528-coated surfaces. The nanopatterned surfaces form uniform and highly ordered nanofingers, with an average length of $68 \pm 3 \mathrm{~nm}$ and width of $24 \pm 2 \mathrm{~nm}$. Platelets seeded on uniform fibrinogen, form fewer and less uniform nanoprotrusions, many of which are long, cone-shaped ( $80 \pm 10$ long, $12 \pm 3 \mathrm{~nm}$ wide).

between the attached filopodia and further extend radially (Figure 3A time point 6, Figure 3B).

It is noteworthy that platelets seeded on uniform fibrinogencoated surfaces display similar spreading properties; with a fraction of platelets arrested at advanced filopodial spreading and a comparable fraction proceeding to full lamellipodial spreading (Figure 4D). The reason for these observations are not well-understood, but the addition of $\mathrm{Mn}^{2+}$ to these platelets, induced a rapid and essentially complete lamellipodial spreading of the platelets that were arrested in the filopodial spreading stage (Figure S1, and Movie S2). This suggests that the transition between the two forms of spreading is highly dependent on the state of $\alpha \mathrm{IIb} \beta 3$ integrin activation. ${ }^{53}$ The fully spread morphology of $\mathrm{Mn}^{2+}$ induced platelets seem to differ from regular spread platelets, the reasons for this is yet to be investigated. Platelet progression through the different phases of spreading differ between fibrinogen and the 30 and $60 \mathrm{~nm}$ surfaces, where we see a faster initialization of spreading on the spaced surfaces, but later, the platelet seems to linger in the lamellipodial stage for a prolonged time (Figure 4D). Monitoring platelets' interaction with surfaces functionalized by $80 / 100$ and $120 \mathrm{~nm}$ ligand spacing could not be visualized by IRM, yet loosely attached platelets could be recognized, mainly by their restricted lateral mobility. As shown in Figure 4B, many of the platelets juxtaposed to the 80 or $100 \mathrm{~nm}$ nanopatterned surfaces extend filopodia, indicating that they were activated
(Movie S3). In contrast, the vast majority of platelets adhering to the $120 \mathrm{~nm}$ surfaces remained discoid (Figure 4C). This observation is consistent with the interpretation that platelets interacting with nanopatterned surfaces with spacing of up to $100 \mathrm{~nm}$ can induce filopodia extension, yet a higher ligand density (spacing of up to $60 \mathrm{~nm}$ ) is needed to further sustain stable adhesion (Figure 4A). In contrast, the nonactivated, discoid platelets attached to the $120 \mathrm{~nm}$ patterns, can sustain a loose adhesion to the substrate.

Given that the spacing between the gold nanoparticles is well below the resolution of conventional light microscopy, ${ }^{54}$ we have utilized high resolution SEM for visualizing of the interface between the platelets and the nanopatterns. The SEM images shown in Figure 5 reveal a platelet, fully spread on the $60 \mathrm{~nm}$ surface, forming clear contacts with the gold particles, mediated by short "nano protrusions" extending from the peripheral lamellipodium (Figure 5). Typical protrusions displayed uniform dimensions in the order of $\sim 25 \mathrm{~nm}$ in diameter and $\sim 70 \mathrm{~nm}$ in length with a variance of $\sim 10 \%$ (Figure 5B,D). The gold particles are best resolved using the BSE mode (Figure $5 \mathrm{~A}, \mathrm{C})$. Occasional local detachment of platelet from the nanopattern provides an opportunity to visualize the ventral surfaces of the flipped-over platelet, revealing multiple protrusions (Figure 6A). These adhesions (Figure 6B,C) had similar dimensions to those formed with uniform fibrinogencoated or gold/SN528-coated glass surfaces (Figure 6C-E and 

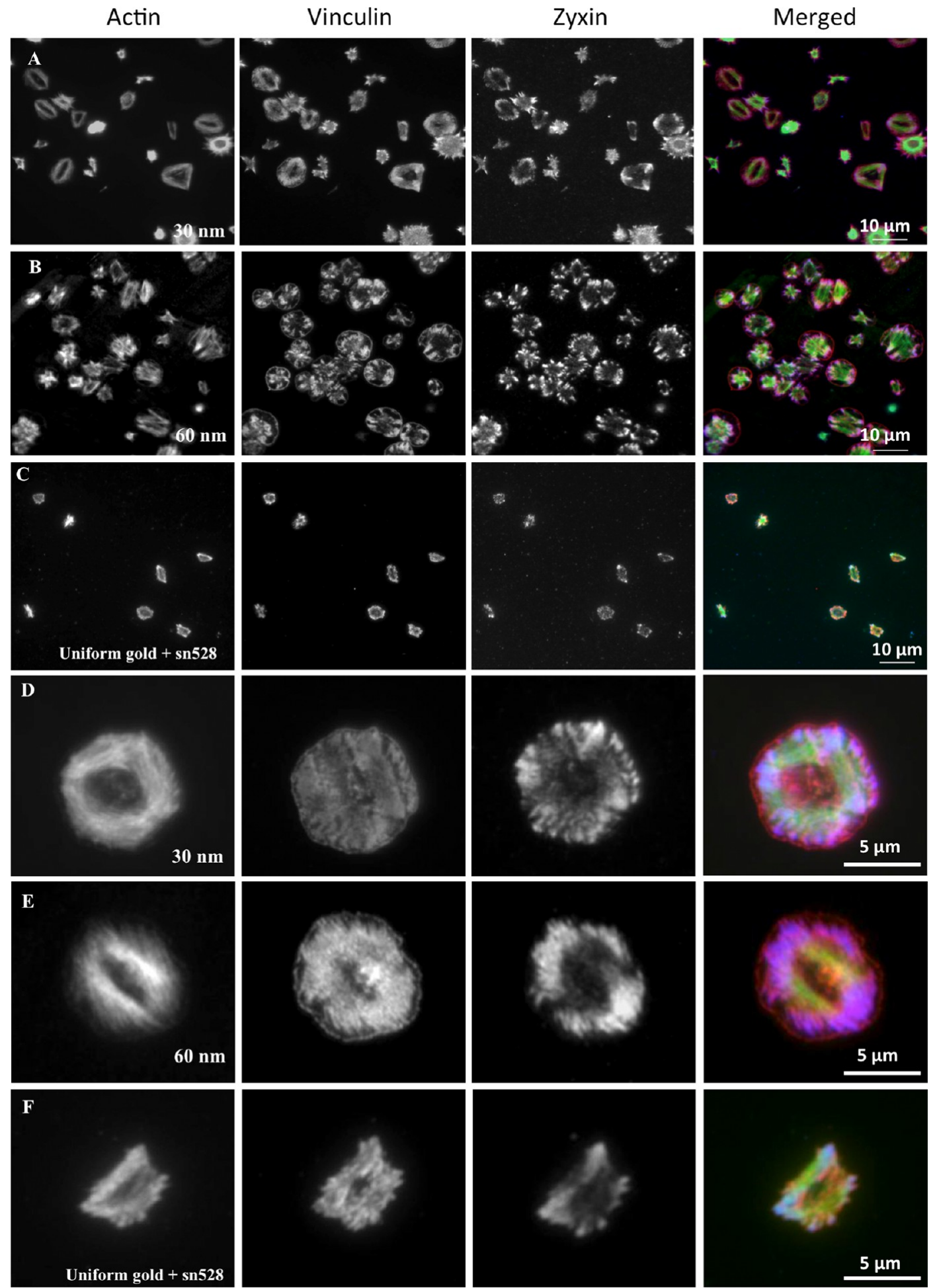

Figure 7. Immunofluorescence images of actin (green), vinculin (red), and zyxin (blue) in platelets, incubation time $1 \mathrm{~h}$, seeded on nanopatterned surface with ligand spacing of (A) $30 \mathrm{~nm}$ (B) $60 \mathrm{~nm}$, (C) uniform gold and SN528. The colocalization in overlapping regions between the 3 proteins appears in magenta at the merged channels. Scale bar: $10 \mu \mathrm{m}$. (D) Platelet on $30 \mathrm{~nm}$ surface demonstrating full spreading. (E) Platelet on $60 \mathrm{~nm}$ surface demonstrating full spreading. (F) Platelet on uniform gold and SN528 surface demonstrating full spreading. Images were taken with a $100 \times$ objective, (scale bar: $5 \mu \mathrm{m}$ ). Rounded shape cells are characterized with radial focal patches, whereas in the polarized cells, focal patches structures localized at the cell's edges.

Figure $5 \mathrm{~B}-\mathrm{D})$, yet the nano protrusions formed on uniform fibrinogen- or gold/SN528-coated surfaces, were less abundant and rather irregular compared to those formed on the nanopatterned surfaces.
To further determine whether ligand spacing on the densely functionalized surfaces, which support platelet spreading (on 30 and $60 \mathrm{~nm}$ pattern), affects the shape and molecular composition of the adhesion sites, we plated platelets on the two adhesive nanopatterned surfaces, fixed them after $60 \mathrm{~min}$, 


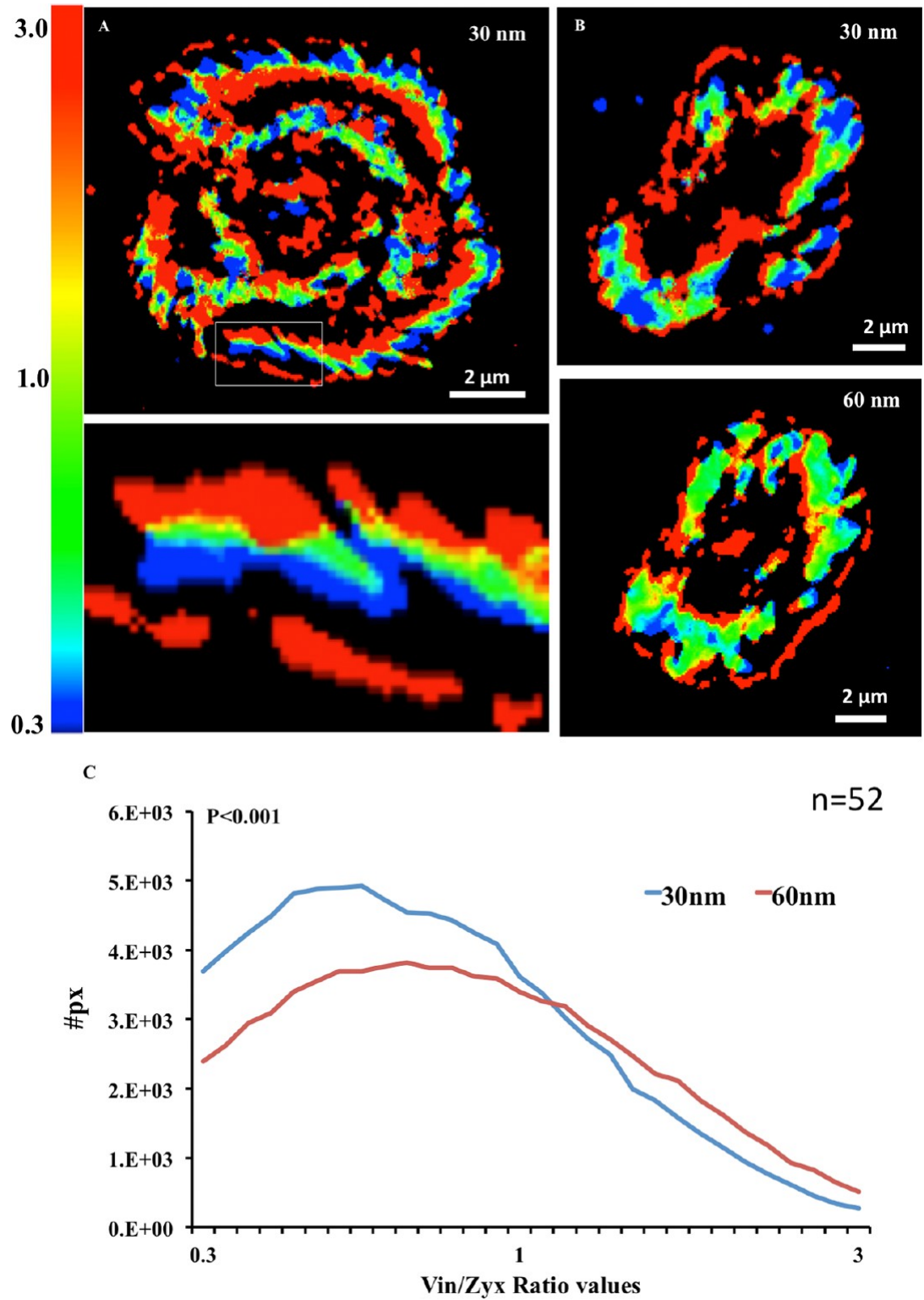

Figure 8. (A top) Ratio imaging (vinculin/zyxin) of platelet on $30 \mathrm{~nm}$ nanopatterned surface, functionalized with SN528. Bottom: expansion of FP area pointing at the distinct pattern of adhesion proteins in focal complex-like structures which are vinculin-rich (red), zyxin-rich (blue), comparable intensity of vinculin and zyxin (green-to-yellow); (B) Ratio images of platelets on $30 \mathrm{~nm}$ (top) and $60 \mathrm{~nm}$ (bottom) nanopatterned surfaces bioactivated with SN528, indicating differences in the adhesion proteins' composition. (C) Summed colored pixels of 27 cells on $30 \mathrm{~nm}$ (blue curve) and $60 \mathrm{~nm}$ (red curve) nanopatterned surfaces, scaled from 0.3 (almost only zyxin) to 3 (almost only vinculin). The sample size is 52 platelets.

and labeled them for F-actin, and the adhesome proteins vinculin and zyxin. Vinculin is a prominent component of essentially all integrin-mediated adhesions, while zyxin is primarily associated with mechanically stimulated integrin adhesions, ${ }^{55,56}$ and thus, the relative levels of the two proteins can shed light on the mechanics of the platelet-surface interaction. As shown in Figure 7A,B, three main morphologies were noted in platelets adhering to the 30 and $60 \mathrm{~nm}$ spacing, respectively; these include (I) poorly spread cells, with compact actin staining and radial filopodia (typical platelet diameter 2-3 $\mu \mathrm{m}$, see Figure $7 \mathrm{~A}, \mathrm{~B})$, (II) Platelets with extensive filopodial spreading, and multiple radial adhesions, containing actin and both vinculin and zyxin. The typical diameter of these platelets was 5-6 $\mu \mathrm{m}$, (Figure 7A,B). (III) Platelets with full lamellipodial spreading and polarized, oval shape with long axis of $7-8 \mu \mathrm{m}$ and minor axis of $5-6 \mu \mathrm{m}$. Surprisingly platelets spread on uniform gold and SN528 show a smaller spread platelet area (Figure $7 \mathrm{C}$ ). In the platelets, actin bundles run at the platelets' flanks, and along the major axis. Vinculin is widely localized at the cell periphery, including focal complexes at the edge of the lamellipodium. Zyxin was absent from the focal complex area and enriched mainly around the platelets' poles (Figure 7D-F). Comparison of the fraction of platelets displaying each morphology on the 30 and $60 \mathrm{~nm}$ surfaces, showed considerable variability, yet, consistently, the spreading on the denser surface $(30 \mathrm{~nm})$ was dominated by full lamellipodial spreading (Figure 7A), while the spreading on the $60 \mathrm{~nm}$ spacing was mostly radial, with multiple filopodia (Figure 7B). To further explore the relative content of vinculin and zyxin in platelets spreading on the two surfaces, we calculated the normalized intensity ratio of vinculin and zyxin. 
The results indicate that zyxin-rich zones (blue) are usually located at the peripheral aspect of the adhesions compared with vinculin-rich contacts, which are detected throughout the ventral membrane of platelets adhering to the $30 \mathrm{~nm}$ surfaces (Figure 8A, showing partial radial spreading and Figure 8B, showing platelet with full lamellipodial spreading). Using the same parameters for calculating the intensity ratios in platelets adhering to the $60 \mathrm{~nm}$ surface showed considerably lower prominence of zyxin-rich adhesions (Figure 8C). Given that recruitment of zyxin is highly contractile force dependent, these results suggest that ligand-dense surfaces, primarily the $30 \mathrm{~nm}$ surface, induce stronger contraction in the attached platelets, which, in turn, induce larger local accumulation of zyxin.

In conclusion, we show, in this study, that variations in adhesive ligand density have multiple effects on platelet activation, surface adhesion and spreading, which have major physiological significance. Specifically, we show that the earliest adhesion-related morphological manifestation of ligand density sensing via integrin $\alpha \mathrm{IIb} \beta 3$ is the extension of filopodia, which, occurs shortly prior to the establishment of stable adhesions with the fibrinogen- or SN528-functionalized matrix. We further show here that a ligand spacing of $100 \mathrm{~nm}$ or less is needed and sufficient for this initial filopodia formation. Once formed, these filopodia can adhere to the functionalized surface-yet the formation of stable adhesions requires higher ligand density (spacing $\leq 60 \mathrm{~nm}$ ), suggesting that the mechanical stability of adhesions requires multiple, close-range molecular interactions. This is consistent with previous reports showing that adhesions of cells to surfaces with interligand spacing of $>70 \mathrm{~nm}$ are highly unstable. ${ }^{41}$

We further show that substrates with ligand spacing of 30 and $60 \mathrm{~nm}$, support platelet adhesion and spreading, comparable to those obtained with platelets seeded on uniform SN528 or fibrinogen, though, the $30 \mathrm{~nm}$ surfaces are more efficient in stimulating full lamellipodial spreading compared to the $60 \mathrm{~nm}$ surfaces, which induced mainly filopodial adhesion and were more effective in recruiting zyxin to the adhesion site, suggesting a higher contractile activity.

The presence of flow would likely affect the adhesion, as adhesion via integrin $\alpha \mathrm{IIb} \beta 3$ is not the primary interaction and adhesion mechanism under shear stress. ${ }^{7}$ Once the primary adhesion is established and the platelets are recruited, we speculate that the inter dot distance would be maintained, but perhaps we would see a faster progression through adhesion stages as the platelets are being activated by the shear stress. ${ }^{57,58}$ Under shear stress, we expect that the platelets attached to the $120 \mathrm{~nm}$ spacing surface will be washed away.

On the 80-100 nm patterns the platelets exert filopodial extension, but are not able to stably attach to the surface (Figure $4 \mathrm{~B})$. The filopodia are straight and the tip of the extension is attached firmly as seen by IRM (Figure 3A time 32'45" black tip of filopodia) suggesting that traction forces are applied to the substrate. $^{59}$ As filopodia are known to be sensitive to spatial cues, ${ }^{60,61}$ on $80-100 \mathrm{~nm}$ surfaces, we observed an extension of filopodia, and then an initial spreading, followed by contraction that leads to detachment as the adhesion to the underlying surface is not strong enough to sustain the adhesion. On lower densities, as the $120 \mathrm{~nm}$ surface, we did not witness filopodial extensions and perhaps therefore the platelets did not retract and detach. The platelets, therefore, remained attached (though in low numbers) as discoid shaped platelets.

A close examination of the interface between the cell membrane and the adhesion ligand, using high-resolution
SEM, indicated that individual membrane adhesions occur via nanoprotrusions that are uniformly attached to the functionalized nanogold particles, both at the lamellipodial periphery and on the filopodial extensions. The regularity of individual adhesions was much higher on the nanopatterned surfaces compared with uniformly functionalized surface, which is attributable to the ligand confinement that induces a highly ordered local molecular interaction pattern and globally regulates the adhesion process.

\section{ASSOCIATED CONTENT}

\section{Supporting Information}

The Supporting Information is available free of charge on the ACS Publications website at DOI: 10.1021/acs.nanolett.8b03513.

Materials and methods, platelets with and without manganese $\left(\mathrm{Mn}^{2+}\right)$, IRM movie showing the adhesion and spreading of platelets on fibrinogen-coated substrate with and without $\mathrm{Mn}^{2+}$, phase contrast movies showing the attachment and spreading of platelets on the nanopatterned surfaces with nanogold spacing of 30,60 , 80, 100, and $120 \mathrm{~nm}$ (PDF)

Movie S1: IRM movie showing the adhesion and spreading of platelets on fibrinogen-coated substrate (AVI)

Movie S2: IRM movie showing the adhesion and spreading of platelets on fibrinogen-coated substrate, with $\mathrm{Mn}^{++}$stimulation at $30 \mathrm{~min}$ time point (AVI)

Movie S3: Phase contrast movies showing the attachment and spreading of platelets on the nano-patterned surfaces with variable nano-gold spacings (AVI)

\section{AUTHOR INFORMATION}

\section{Corresponding Author}

*E-mail: benny.geiger@weizmann.ac.il. Tel.: +972-52 3488848. Fax: +972-8-946 5607 .

ORCID

Horst Kessler: 0000-0002-7292-9789

Joachim P. Spatz: 0000-0003-3419-9807

\section{Author Contributions}

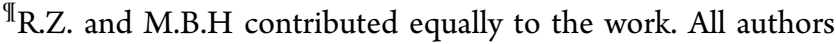
contributed to the planning of the project and writing the manuscript. All authors have given approval to the final version of the manuscript.

\section{Notes}

The authors declare no competing financial interest.

\section{ACKNOWLEDGMENTS}

We would like to express our gratitude to the European Research Council (ERC) for supporting this project (ERC Advanced Grant under grant agreement no. 294852-SynAd (to B.G. and J.S.). This research was supported by a grant from the Ministry of Science, Technology \& Space, Israel \& the Ministry of Foreign Affairs and the Ministry of Higher Education and Research of France, Grant no. 3-13024 (to B.G.). H.K. thanks the Deutsche Forschungsgemeinschaft and the Max Planck Society for their financial support (CIPSM). The authors are grateful to Barbara Morgenstern for her expert help in the style editing of this manuscript. B.G. is the Incumbent of The Erwin Neter Professorial Chair in Cell and Tumor Biology. 


\section{REFERENCES}

(1) Thon, J. N.; Italiano, J. E. Handb. Exp. Pharmacol. 2012, 210, 322.

(2) Machlus, K. R.; Italiano, J. E., Jr. J. Cell Biol. 2013, 201 (6), 78596.

(3) Menter, D. G.; Tucker, S. C.; Kopetz, S.; Sood, A. K.; Crissman, J.

D.; Honn, K. V. Cancer Metastasis Rev. 2014, 33 (1), 231-69.

(4) Ruggeri, Z. M. Nat. Med. 2002, 8 (11), 1227-34.

(5) Varga-Szabo, D.; Pleines, I.; Nieswandt, B. Arterioscler., Thromb., Vasc. Biol. 2008, 28 (3), 403-12.

(6) Li, Z.; Delaney, M. K.; O’Brien, K. A.; Du, X. Arterioscler., Thromb., Vasc. Biol. 2010, 30 (12), 2341-9.

(7) Ruggeri, Z. M. Microcirculation 2009, 16 (1), 58-83.

(8) Ruggeri, Z. M.; Mendolicchio, G. L. Circ. Res. 2007, 100 (12), 1673-85.

(9) Shattil, S. J.; Newman, P. J. Blood 2004, 104 (6), 1606-15.

(10) Mendelboum Raviv, S.; Szekeres-Csiki, K.; Jenei, A.; Nagy, J.; Shenkman, B.; Savion, N.; Harsfalvi, J. Thromb. Res. 2012, 129 (4), e29-35.

(11) Nieswandt, B.; Brakebusch, C.; Bergmeier, W.; Schulte, V.; Bouvard, D.; Mokhtari-Nejad, R.; Lindhout, T.; Heemskerk, J. W.; Zirngibl, H.; Fassler, R. EMBO J. 2001, 20 (9), 2120-30.

(12) Pugh, N.; Simpson, A. M.; Smethurst, P. A.; de Groot, P. G.; Raynal, N.; Farndale, R. W. Blood 2010, 115 (24), 5069-79.

(13) Siljander, P. R.; Munnix, I. C.; Smethurst, P. A.; Deckmyn, H.; Lindhout, T.; Ouwehand, W. H.; Farndale, R. W.; Heemskerk, J. W. Blood 2003, 103 (4), 1333-1341.

(14) Vanhoorelbeke, K.; Ulrichts, H.; Schoolmeester, A.; Deckmyn, H. Curr. Drug Targets: Cardiovasc. \&amp; Haematol. Disord. 2003, 3 (2), 125-40.

(15) Hartwig, J. H. J. Cell Biol. 1992, 118 (6), 1421-42.

(16) Moroi, M.; Jung, S. M. Front. Biosci., Landmark Ed. 1998, 3, d719-28.

(17) Sorrentino, S.; Studt, J. D.; Medalia, O.; Tanuj Sapra, K. Eur. J. Cell Biol. 2015, 94 (3-4), 129-38.

(18) Poulter, N. S.; Pollitt, A. Y.; Davies, A.; Malinova, D.; Nash, G. B.; Hannon, M. J.; Pikramenou, Z.; Rappoport, J. Z.; Hartwig, J. H.; Owen, D. M.; Thrasher, A. J.; Watson, S. P.; Thomas, S. G. Nat. Commun. 2015, 6, 7254.

(19) Jirouskova, M.; Jaiswal, J. K.; Coller, B. S. Blood 2007, 109 (12), 5260-9.

(20) Naik, M. U.; Naik, U. P. Blood 2003, 102 (10), 3629-36.

(21) Bearer, E. L.; Prakash, J. M.; Li, Z. Int. Rev. Cytol. 2002, 217, 13782.

(22) Hartwig, J. H. The Platelet Cytoskeleton. In Platelets, 3rd ed.; Michelson, A. D., Ed.; Academic Press: San Diego, CA, 2013; pp 145168.

(23) McCarty, O. J.; Larson, M. K.; Auger, J. M.; Kalia, N.; Atkinson, B. T.; Pearce, A. C.; Ruf, S.; Henderson, R. B.; Tybulewicz, V. L.; Machesky, L. M.; Watson, S. P. J. Biol. Chem. 2005, 280 (47), 3947484.

(24) Thornber, K.; McCarty, O. J.; Watson, S. P.; Pears, C. J. FEBS J. 2006, 273 (22), 5032-43.

(25) Leng, L.; Kashiwagi, H.; Ren, X. D.; Shattil, S. J. Blood 1998, 91 (11), 4206-4215.

(26) Calaminus, S. D.; Thomas, S.; McCarty, O. J.; Machesky, L. M.; Watson, S. P. J. Thromb. Haemostasis 2008, 6 (11), 1944-52.

(27) Bershadsky, A. D.; Balaban, N. Q.; Geiger, B. Annu. Rev. Cell Dev. Biol. 2003, 19, 677-95.

(28) Geiger, B.; Bershadsky, A. Cell 2002, 110 (2), 139-42.

(29) Janmey, P. A.; Weitz, D. A. Trends Biochem. Sci. 2004, 29 (7), 364-70.

(30) Orr, A. W.; Helmke, B. P.; Blackman, B. R.; Schwartz, M. A. Dev. Cell 2006, 10 (1), 11-20.

(31) Lam, W. A.; Chaudhuri, O.; Crow, A.; Webster, K. D.; Li, T. D.; Kita, A.; Huang, J.; Fletcher, D. A. Nat. Mater. 2011, 10 (1), 61-6.

(32) Liang, X. M.; Han, S. J.; Reems, J. A.; Gao, D.; Sniadecki, N. J. Lab Chip 2010, 10 (8), 991-8.
(33) Discher, D. E.; Janmey, P.; Wang, Y. L. Science 2005, 310 (5751), 1139-43.

(34) Georges, P. C.; Janmey, P. A. J. Appl. Physiol. 2005, 98 (4), $1547-$ 53.

(35) Pelham, R. J., Jr.; Wang, Y. Proc. Natl. Acad. Sci. U. S. A. 1997, 94 (25), 13661-5.

(36) Wells, R. G. Hepatology 2008, 47 (4), 1394-400.

(37) Dalby, M. J.; Childs, S.; Riehle, M. O.; Johnstone, H. J.; Affrossman, S.; Curtis, A. S. Biomaterials 2003, 24 (6), 927-35.

(38) Dalby, M. J.; Yarwood, S. J.; Riehle, M. O.; Johnstone, H. J.; Affrossman, S.; Curtis, A. S. Exp. Cell Res. 2002, 276 (1), 1-9.

(39) Ebendal, T. Exp. Cell Res. 1976, 98 (1), 159-69.

(40) Jungbauer, S.; Kemkemer, R.; Gruler, H.; Kaufmann, D.; Spatz, J. P. ChemPhysChem 2004, 5 (1), 85-92.

(41) Arnold, M.; Cavalcanti-Adam, E. A.; Glass, R.; Blummel, J.; Eck, W.; Kantlehner, M.; Kessler, H.; Spatz, J. P. ChemPhysChem 2004, 5 (3), 383-8.

(42) Arnold, M.; Hirschfeld-Warneken, V. C.; Lohmuller, T.; Heil, P.; Blummel, J.; Cavalcanti-Adam, E. A.; Lopez-Garcia, M.; Walther, P.; Kessler, H.; Geiger, B.; Spatz, J. P. Nano Lett. 2008, 8 (7), 2063-9.

(43) Cavalcanti-Adam, E. A.; Volberg, T.; Micoulet, A.; Kessler, H.; Geiger, B.; Spatz, J. P. Biophys. J. 2007, 92 (8), 2964-74.

(44) Cavalcanti-Adam, E. A.; Aydin, D.; Hirschfeld-Warneken, V. C.; Spatz, J. P. HFSP J. 2008, 2 (5), 276-85.

(45) Ekerdt, B. L.; Segalman, R. A.; Schaffer, D. V. Biotechnol. J. 2013, 8 (12), 1411-23.

(46) Deeg, J. A.; Louban, I.; Aydin, D.; Selhuber-Unkel, C.; Kessler, H.; Spatz, J. P. Nano Lett. 2011, 11 (4), 1469-76.

(47) Selhuber-Unkel, C.; Erdmann, T.; Lopez-Garcia, M.; Kessler, H.; Schwarz, U. S.; Spatz, J. P. Biophys. J. 2010, 98 (4), 543-51.

(48) Deng, J.; Zhao, C.; Spatz, J. P.; Wei, Q. ACS Nano 2017, 11 (8), $8282-8291$.

(49) Oria, R.; Wiegand, T.; Escribano, J.; Elosegui-Artola, A.; Uriarte, J. J.; Moreno-Pulido, C.; Platzman, I.; Delcanale, P.; Albertazzi, L.; Navajas, D.; Trepat, X.; Garcia-Aznar, J. M.; Cavalcanti-Adam, E. A.; Roca-Cusachs, P. Nature 2017, 552 (7684), 219-224.

(50) Frohnmayer, J. P.; Bruggemann, D.; Eberhard, C.; Neubauer, S.; Mollenhauer, C.; Boehm, H.; Kessler, H.; Geiger, B.; Spatz, J. P. Angew. Chem., Int. Ed. 2015, 54 (42), 12472-8.

(51) Hook, K. M.; Bennett, J. S. Handb. Exp. Pharmacol. 2012, 210, 199-223.

(52) Bollinger, M.; Manzenrieder, F.; Kolb, R.; Bochen, A.; Neubauer, S.; Marinelli, L.; Limongelli, V.; Novellino, E.; Moessmer, G.; Pell, R.; Lindner, W.; Fanous, J.; Hoffman, A.; Kessler, H. J. Med. Chem. 2012, $55(2), 871-82$.

(53) Yan, B.; Hu, D. D.; Knowles, S. K.; Smith, J. W. J. Biol. Chem. 2000, 275 (10), 7249-60.

(54) Spatz, J. P.; Geiger, B. Methods Cell Biol. 2007, 83, 89-111.

(55) Lele, T. P.; Pendse, J.; Kumar, S.; Salanga, M.; Karavitis, J.; Ingber, D. E. J. Cell. Physiol. 2006, 207 (1), 187-94.

(56) Hirata, H.; Tatsumi, H.; Sokabe, M. Commun. Integr. Biol. 2008, 1 (2), 192-5.

(57) Dunkley, S.; Harrison, P. Platelets 2005, 16 (2), 81-4.

(58) Lu, Q.; Hofferbert, B. V.; Koo, G.; Malinauskas, R. A. Artif. Organs 2013, 37 (10), 894-903.

(59) Albuschies, J.; Vogel, V. Sci. Rep. 2013, 3, 1658.

(60) Kita, A.; Sakurai, Y.; Myers, D. R.; Rounsevell, R.; Huang, J. N.; Seok, T. J.; Yu, K.; Wu, M. C.; Fletcher, D. A.; Lam, W. A. PLoS One 2011, 6 (10), No. e26437.

(61) Sandmann, R.; Koster, S. Sci. Rep. 2016, 6, 22357. 